\title{
Efecto del aumento de la temperatura en la fotosíntesis de una especie alto-andina en dos altitudes
}

\section{Effect of the increase in temperature in the photosynthesis of a high-andean species at two elevations}

\author{
Camila Sanfuentes ${ }^{1,2}$, Angela Sierra-Almeida ${ }^{1,2 *}$ \& Lohengrin A. Cavieres ${ }^{1,2}$ \\ 1Departamento de Botánica, Facultad de Ciencias Naturales y Oceanográficas, Universidad de Concepción, Casilla 160-C, \\ Concepción, Chile. \\ ${ }^{2}$ Instituto de Ecología y Biodiversidad (IEB), Casilla 653, Santiago, Chile. \\ *angelasierra@udec.cl; a.sierra.almeida@gmail.com
}

\section{RESUMEN}

Se ha propuesto que los ambientes de alta montaña son especialmente vulnerables al calentamiento global. La interacción de variables como déficit hídrico y las condiciones de temperatura ambiental puede restringir el intercambio gaseoso en zonas de alta-montaña. Debido a las distintas limitaciones abióticas que se encuentran en un gradiente altitudinal, se propone que el aumento de la temperatura afectará positivamente la fotosíntesis de la planta alto-andina Phacelia secunda en elevadas altitudes y negativamente a las plantas de bajas altitudes. En el presente estudio se evaluó el efecto del aumento de la temperatura ambiental sobre el intercambio gaseoso de $P$. secunda en dos altitudes: $2900 \mathrm{~m}$ y $3600 \mathrm{~m}$. En cada altitud se expusieron seis individuos de $P$. secunda a un sistema de calentamiento pasivo denominado "Open Top Chamber" (OTC) que aumenta la temperatura del aire en $c a .3^{\circ} \mathrm{C}$. Adicionalmente, en cada altitud se seleccionaron otros seis individuos en espacios abiertos para utilizarlos como control y que se encontraban a $2 \mathrm{~m}$ de la OTC más cercana. En cada individuo se midió el intercambio gaseoso y el potencial hídrico xilemático. La temperatura ambiental fue en promedio $3,5^{\circ} \mathrm{C}$ mayor dentro de las OTC en ambas altitudes. En contraste, el potencial hídrico del suelo y de las plantas se redujo un $28 \%$ al interior de las OTC, pero sólo a $2900 \mathrm{~m}$. En esta altitud, las tasas de fotosíntesis al interior de las OTC se redujeron un $40,7 \%$, mientras que a $3600 \mathrm{~m}$ las tasas aumentaron un $24,4 \%$. Los efectos diferenciales en las tasas de fotosíntesis debido a la temperatura fueron acompañados con cambios en la conductancia estomática. Esto sugiere que los efectos del calentamiento global sobre la fotosíntesis de $P$. secunda son contrastantes entre ambas altitudes debido a los efectos secundarios que éste tiene sobre la disponibilidad hídrica en cada altitud.

Palabras clave: Phacelia secunda, calentamiento global, estrés por temperatura, sequía, conductancia estomática.

\begin{abstract}
High-alpine environments have been proposed as particularly vulnerable to global warming. The interaction of variables such as water deficit and temperature conditions may restrict gas exchange in high-alpine areas. Due to different abiotic constraints that occur at different elevations in the Andes of central Chile we hypothesize that the temperature increases will positively affect the photosynthesis of the high-Andean plant Phacelia secunda at high elevation but it will be negatively affected by warming at lower elevations. In this study we evaluated the effect of increased environmental temperature on the gas exchange of $P$. secunda at two contrasting elevations: $2900 \mathrm{~m}$ and $3600 \mathrm{~m}$ a.s.l. At each elevation we exposed six individuals of $P$. secunda to a passive warming system with "Open Top Chamber" (OTC) that increased air temperature on ca. $3^{\circ} \mathrm{C}$. Other six individuals we selected in open areas and maintained as control on each elevation. At both elevations, on each selected individual (i.e. within and outside OTCs) we measured gas exchange and xylem water potential. At both elevations air temperature was on average $3.5^{\circ} \mathrm{C}$ higher inside the OTC. In contrast, OTC reduced $28 \%$ the soil and xylem water potential only at $2900 \mathrm{~m}$. The increased temperature inside the OTC reduced $40.7 \%$ gas exchange rates at $2900 \mathrm{~m}$, but increased it $24.4 \%$ at $3600 \mathrm{~m}$. These differential effects of warming on photosynthetic rates were accompanied by changes in stomatal conductance. This suggests that the effects of global warming on the photosynthesis of $P$. secunda at two contrasting altitudes are related with the concomitant changes on drought at each elevation.
\end{abstract}

KeYwords: Phacelia secunda, global warming, temperature stress, drought, stomatal conductance. 


\section{INTRODUCCIÓN}

Durante los últimos siglos se han observado una serie de cambios en las condiciones climáticas generales del planeta, fenómeno que ha sido denominado "cambio climático global" (Walther 2003). Uno de los cambios más estudiado y más documentado es el aumento en la temperatura, encontrándose que durante los últimos 30 años la temperatura media del planeta ha aumentado entre $0,5^{\circ} \mathrm{y}$ $3^{\circ} \mathrm{C}$ (IPCC 2007). A su vez, diversos modelos predicen que en los próximos 50 años la temperatura global del planeta podría incrementarse entre $1,3^{\circ}$ a $5,8^{\circ} \mathrm{C}$ comparado con tiempos pre-industriales (IPCC 2007). Si bien este aumento global de la temperatura afectará a todos los ecosistemas del planeta, se ha establecido que algunos de ellos serán más sensibles que otros a dichas modificaciones climáticas (Walther et al. 2002, Nemani et al. 2003). Al respecto, se ha sugerido que entre los ecosistemas más sensibles al cambio climático global están los hábitats de alta latitud (tundras árticas y antárticas) y alta altitud (hábitats de altamontaña) (Körner 2000, 2003). De hecho, varios estudios han documentado importantes cambios en las temperaturas de dichos hábitats durante los últimos siglos (ver revisiones de Beniston et al. 1997 y Díaz \& Bradley 1997).

Los ambientes de alta montaña son aquellos que se encuentran por sobre el límite altitudinal de crecimiento de los árboles (Körner 2003) y se caracterizan por presentar condiciones climáticas muy estresantes para la supervivencia y reproducción de las plantas (Billings \& Mooney 1968, Bliss 1971, Billings 1974, Körner 2003). Las bajas temperaturas del aire y del suelo, altos niveles de radiación solar, fuertes vientos, corta duración de la estación de crecimiento y escasez de nutrientes son las principales características de estos hábitats (Bliss 1985, Körner 2003). Dado que en estos ambientes los factores abióticos anteriormente mencionados son los principales limitantes para el crecimiento y supervivencia de las plantas (Körner 2003), cualquier modificación de éstos producto del cambio climático global tendrá importantes consecuencias sobre el desempeño fisiológico de las mismas (Körner 2003, Nagy \& Grabherr 2009).

Estudios previos han demostrado que en muchas de las plantas que habitan zonas de alta-montaña el proceso fotosintético es altamente eficiente a bajas temperaturas y a una alta radiación (Körner \& Larcher 1988, Streb et al. 1998, Germino \& Smith 2001). En términos de capacidad fotosintética, las plantas de alta montaña no se diferencian de plantas de otros tipos de ambientes más benignos (Körner 2003). No obstante, se ha observado que las temperaturas a las cuales se realiza la máxima tasa de fotosíntesis (i.e. óptimo de fotosíntesis) se relacionan con las temperaturas foliares que se alcanzan en los períodos de máxima radiación (Körner 2003). Dada la gran cantidad de días nublados que se dan a elevadas altitudes durante la época libre de nieve (Körner 2003), se ha sugerido que una de las principales limitaciones para mantener un balance de carbono positivo en estos ambientes es la densidad de flujo fotónico (Körner \& Diemer 1987, Körner 2003), ya que las especies estarían menor cantidad de tiempo realizando sus máximos de fotosíntesis. Por lo tanto, si bien la temperatura per se no es una limitante de la fotosíntesis (Körner 2003), la cantidad de tiempo con temperaturas cercanas al óptimo fotosintético pueden ser un factor clave en determinar los efectos que tendría el calentamiento global sobre el desempeño fotosintético de las plantas de alta-montaña.

Aunque durante las últimas décadas se han llevado a cabo varios estudios que han evaluado los efectos del aumento de la temperatura en ecosistemas de altamontaña, las respuestas más comúnmente evaluadas han sido tasa de crecimiento, cambio en biomasa, esfuerzo y éxito reproductivo, productividad, etc. (ver revisiones de Henry \& Molau 1997, Arft et al. 1999, Rustad et al. 2001, Dormann \& Woodin 2002, Walker et al. 2006). Pocos estudios han evaluado las respuestas fotosintéticas de especies de alta-montaña al aumento de la temperatura, encontrándose evidencias contradictorias entre ellos. Por ejemplo, mientras en algunos casos se encontraron aumentos en las tasas de fotosíntesis con el incremento de la temperatura ambiental (Welker et al. 1999, 2004), en otros no se observaron diferencias (Hobbie \& Chapin 1998). Welker et al. (2004) sugieren que tales diferencias en las respuestas fotosintéticas al aumento de la temperatura estarían determinadas por la co-variación en la disponibilidad hídrica del suelo. En tundras xéricas, por ejemplo, el aumento en la temperatura disminuiría la disponibilidad hídrica, lo cual desencadenaría menores niveles de conductancia estomática y una disminución en la asimilación de $\mathrm{CO}_{2}$. Körner (2003) considera que en general la disponibilidad hídrica no es un factor limitante para la biología de las plantas de alta-montaña. Sin embargo, varios estudios demuestran que la disponibilidad de agua en el suelo es un factor clave en el desempeño fisiológico de las plantas en zonas de alta-montaña con influencia de clima tipo mediterráneo, donde los veranos son muy secos (e.g. Loik \& Redar 2003, Loik et al. 2004, Sierra-Almeida et al. 2009). Por ejemplo, Loik et al. (2000) encontraron que la conductancia estomática y la tasa de fotosíntesis de Erigeron speciosus y Artemisia tridentata disminuyeron con el aumento de la temperatura, el que a su vez produjo una mayor desecación del suelo.

Los Andes de Chile central se caracterizan por presentar una estación de crecimiento (período libre de nieve) seca que limita fuertemente el establecimiento de plántulas (Cavieres et al. 2006, 2007). No obstante, se ha encontrado que las limitaciones hídricas disminuyen a medida que aumentamos en altitud (Cavieres et al. 2006), siendo las limitaciones por bajas temperaturas las que comienzan a tomar mayor importancia a mayores altitudes (Cavieres et al. 2007). Si 
en bajas altitudes (caracterizadas por una marcada sequía estival) se aumenta la temperatura ambiental, entonces habrán mayores demandas evaporativas tanto para las plantas como para el suelo, tornando el ambiente aún más seco. Esto sugiere que futuros aumentos en la temperatura tornarían los ambientes de altitudes bajas mucho más limitantes para el desempeño fotosintético de las plantas. En contraste, en altitudes mayores donde la principal limitante para la fotosíntesis ya no es la disponibilidad hídrica en el suelo sino la baja temperatura, el calentamiento global podría tener consecuencias positivas para la fotosíntesis.

En el presente estudio evaluamos las posibles consecuencias del aumento de la temperatura ambiental sobre la fotosíntesis de plantas de alta-montaña, sometiendo en forma experimental a individuos de la especie Phacelia secunda J.F. Gmel. (Boraginaceae) a un incremento de la temperatura ambiental en dos altitudes durante una estación de crecimiento. P. secunda es una hierba perenne que en Chile presenta una extraordinaria amplitud tanto en su distribución latitudinal como altitudinal, lo cual la hace un buen modelo de estudio para evaluar cambios en variables fisiológicas en respuesta a los cambios ambientales, controlando en gran medida los efectos genotípicos.

\section{MATERIALES Y MÉTODOS}

Sitio de ESTUdio

El área de estudio corresponde a la zona de alta montaña de la Cordillera de los Andes de Chile central ( $\left.33^{\circ} \mathrm{S}\right)$, distante $50 \mathrm{~km}$ de la ciudad de Santiago. Se eligieron dos sitios de estudio, los cuales presentan características mesoclimáticas contrastantes (Cavieres et al. 2007). El primer sitio está ubicado a $2900 \mathrm{~m}$ sobre el nivel del mar, en una ladera de exposición norte, cercana al centro de Ski La Parva $\left(33^{\circ} 21^{\prime} \mathrm{S}, 70^{\circ} 19^{\prime} \mathrm{W}\right)$. El otro sitio está ubicado a $3600 \mathrm{~m}$ de altitud, en una ladera de exposición noreste $\left(33^{\circ} 19^{\prime} \mathrm{S}\right.$, $\left.70^{\circ} 15^{\prime} \mathrm{W}\right)$. Mientras a $2900 \mathrm{~m}$ la estación de crecimiento comienza habitualmente en octubre y termina en abril, a $3600 \mathrm{~m}$ la estación de crecimiento comienza en diciembre y finaliza en marzo. La temperatura media del aire durante la estación de crecimiento es de $10,9^{\circ} \mathrm{C}$ y $6,8^{\circ} \mathrm{C}$ a 2900 y 3600 $\mathrm{m}$ de altitud, respectivamente (Cavieres et al. 2007).

Las precipitaciones en los Andes de Chile central ocurren principalmente en forma de nieve durante el invierno. Los veranos son secos, con lluvias ocasionales, las cuales son más frecuentes a mayor altitud (Santibáñez \& Uribe 1990). Ambos sitios presentan diferencias en la disponibilidad hídrica del suelo. Mientras a $3600 \mathrm{~m}$ el potencial hídrico del suelo se mantiene cercano a $-0.3 \mathrm{MPa}$ durante toda la estación de crecimiento, a $2900 \mathrm{~m}$ el potencial hídrico del suelo disminuye de -1.6 MPa en noviembre a -4.5 $\mathrm{MPa}$ en marzo (Sierra-Almeida et al. 2009).

\section{ESPECIE ESTUDIADA}

Phacelia secunda J.F.Gmel. (Boraginaceae) es una hierba perenne que en Chile presenta una extraordinaria amplitud tanto en su distribución latitudinal como altitudinal. Su distribución abarca desde Parinacota $\left(18^{\circ} \mathrm{S}\right)$ hasta Tierra del Fuego (54 $\mathrm{S}$ ) y desde el nivel del mar hasta cerca del límite altitudinal de la vegetación andina (3600 m) (Cavieres 2000). Dependiendo de la altitud las plantas son arrosetadas o con tallos erguidos, con rizoma vertical. Los tallos presentan pilosidad argenteo-serícea, adpresa, en menor medida con pilosidad hirsuta. Las hojas basales son largamente pecioladas, las caulinares casi sésiles, pilosas en ambas caras. Las inflorescencias son cimas escorpioides, laxas o contraídas. El cáliz es de igual o menor longitud que la corola, los sépalos son más o menos lanceolados, hirsutos. La corola es campanulada, de color lila a blanco. Los estambres son exertos. El fruto es una cápsula piriforme, que contiene entre 1-4 semillas. Las semillas son naviculares, de 2-3 $\mathrm{mm}$ de longitud por $1 \mathrm{~mm}$ de ancho; tienen un extremo marcadamente agudo, foveoladas en ambas caras y son de color castaño oscuro.

En general, las especies que crecen en un amplio rango altitudinal deben adaptarse a distintas condiciones, tales como la disminución de la temperatura del aire y del suelo, al aumento de los niveles de radiación y velocidad del viento y a la disminución de la duración de la estación de crecimiento (Körner 2003). Phacelia secunda presenta una marcada variación altitudinal en su morfología, desde plantas con rosetas pequeñas, acaulescentes, de hojas erectas, con uno o dos tallos florales altos a baja altitud hasta plantas con rosetas grandes, con hojas y tallos florales cortos que crecen a ras de suelo (Cavieres 2000).

\section{DiSEÑO EXPERIMENTAL}

En cada altitud se seleccionaron 12 individuos de Phacelia secunda de tamaño similar y visualmente sanas. En cada sitio, 6 de estos individuos fueron asignados aleatoriamente a un tratamiento de aumento de temperatura ambiental. Para esto se utilizó un sistema de calentamiento pasivo denominado OTC (Open Top Chamber), basado en el protocolo estandarizado de la red científica ITEX (Internacional Tundra Experiment: http://www.geog.ubc. ca/itex/). Las OTC han sido ampliamente utilizadas en el estudio de las respuestas de las plantas al cambio climático en ecosistemas árticos, subárticos y de alta-montaña, incrementando la temperatura del aire entre 2 y $4^{\circ} \mathrm{C}$ (Henry \& Molau 1997, Norby et al. 1997, Aerts et al. 2004, Ren et al. 2010). Los otros 6 individuos se ubicaron en espacios abiertos (EA), a unos $2 \mathrm{~m}$ de la OTC más cercana y fueron utilizados como control. Los individuos seleccionados para este estudio llevaban 3 temporadas creciendo en las condiciones experimentales antes descritas. Los cambios en temperatura y potencial hídrico del suelo producidos por 
las OTC fueron consistentes entre dichas temporadas y se detallan en la Tabla I.

En cada uno de los individuos seleccionados, i.e. plantas de espacio abierto (EA) y en (OTC) se midió el intercambio gaseoso y potencial hídrico xilemático al final del período de crecimiento ( 27 y 28 de marzo de 2009), que es cuando se observan las mayores diferencias altitudinales en temperatura y disponibilidad hídrica (Sierra-Almeida et al. 2009). Las mediciones se realizaron entre las 12:00 y 14:00 h, que es cuando se registran los más altos niveles de radiación y temperatura en ambas altitudes.

\section{PotenCIAL hídRICO DEL SUELO Y XILEMÁTICO}

En ambas altitudes, el potencial hídrico del suelo $\left(\mathrm{\Psi H}_{2} \mathrm{O}_{\text {suelo }}\right)$ fue medido en cada condición experimental (i.e. EA y OTC) utilizando psicrómetros PST-55 (Wescor, USA). A 2900 m, 3 psicrómetros por condición fueron enterrados a una profundidad de $50 \mathrm{~cm}$. A $3600 \mathrm{~m}$, los 3 psicrómetros se enterraron a una profundidad de $30 \mathrm{~cm}$. En ambos casos, los psicrómetros se ubicaron en la zona del suelo donde se concentra la mayor parte de la biomasa radicular. Los datos de $\Psi_{2} \mathrm{O}_{\text {suelo }}$ se obtuvieron conectando los psicrómetros a un data-logger Psypro (Wescor, USA).

En cada altitud se midió el potencial hídrico xilemático $\left(\mathrm{\Psi H}_{2} \mathrm{O}_{\text {xilemático }}\right)$ en las plantas de Phacelia secunda creciendo en cada condición experimental (i.e. 6 individuos por condición). De cada individuo se extrajo una hoja, totalmente expandida, de una misma altura dentro de la roseta y similar exposición lumínica. Las mediciones de $\Psi_{2} \mathrm{O}_{2}$ xilemático realizaron con una bomba Scholander (PMS Instrument Company, USA).

\section{INTERCAMBIO DE GASES}

En cada altitud y condición experimental se seleccionó una hoja por individuo con los mismos criterios utilizados en la medición de $\Psi_{2} \mathrm{O}_{\text {xilemático }}$. En cada hoja se midió la tasa de intercambio gaseoso utilizando un analizador de gas infrarrojo IRGA (CIRAS-2, PP-Systems, USA). Las mediciones se realizaron a una concentración constante de $\mathrm{CO}_{2}$ de $370 \mu \mathrm{mol} \mathrm{mol}^{-1}$ y $40 \%$ de humedad relativa. Tanto la temperatura como la radiación correspondieron a los registrados naturalmente a la hora de las mediciones, en los mismos días que se realizaron las mediciones de $\Psi \mathrm{H} 2 \mathrm{O}$.

Las variables de intercambio de gases consideradas fueron: An, tasa de asimilación neta $\left(\mu \mathrm{mol} \mathrm{CO}_{2} \mathrm{~m}^{-2} \mathrm{~s}^{-1}\right)$; Gs, conductancia estomática $\left(\mathrm{mmol} \mathrm{m} \mathrm{m}^{-2} \mathrm{~s}^{-1}\right) \mathrm{y} \mathrm{E}$, transpiración $\left(\mu \mathrm{mol} \mathrm{H}_{2} \mathrm{O} \mathrm{m}^{-2} \mathrm{~s}^{-1}\right.$ ). Dado que no todas las hojas cubrían completamente el área de la cubeta del IRGA, cada hoja fue fotografiada dentro de la cubeta y se calculó su área utilizando un software procesador de imágenes. Con esta información, todas las variables antes descritas fueron corregidas por área foliar, de modo de estandarizar todas las mediciones a un área de $2,5 \mathrm{~cm}^{2}$, que es el área de la cubeta del IRGA utilizada en las mediciones. El uso eficiente del agua (UEA), que es la razón entre la ganancia de $\mathrm{CO}_{2}$ por fotosíntesis y la pérdida de agua por transpiración se calculó como UEA $=$ An/E de acuerdo a lo propuesto por Lambers et al. (2008).

TABLA I. Modificaciones microclimáticas producidas por la instalación de OTCs en dos altitudes de Los Andes de Chile central durante 3 temporadas de crecimiento: diciembre-abril para $2900 \mathrm{~m}$ y diciembre-marzo para $3600 \mathrm{~m}$ de altitud. La temperatura media corresponde al promedio para toda la temporada de crecimiento \pm EE. El potencial hídrico del suelo $\left(\Psi_{2} \mathrm{H}_{\text {suelo }}\right)$ corresponde al promedio diario en marzo o abril según la altitud $(\mathrm{n}=5, \pm \mathrm{EE})$. Letras en superíndice distintas indican diferencias significativas entre condiciones experimentales (Prueba de $t ; p<0,05$ )

TABLE I. Microclimatic modifications produced by OTCs at two elevations in the central Chilean Andes during 3 growing seasons: December-April for $2900 \mathrm{~m}$ and December-March for $3600 \mathrm{~m}$. Air temperature values correspond to mean growing season \pm SE. Soil water potential $\left(\mathrm{\Psi H}_{2} \mathrm{O}_{\text {suelo }}\right)$ corresponds to daily mean values for march or april depending on elevation $(\mathrm{n}=5$, $\pm \mathrm{SE})$. Upper-case letters indicate significant differences between experimental conditions $(t$ test; $p<0.05)$.

\begin{tabular}{lcccc}
\hline & \multicolumn{2}{c}{$2900 \mathrm{~m}$} & \multicolumn{2}{c}{$3600 \mathrm{~m}$} \\
\cline { 2 - 4 } TEMPORADA 2006-2007 & EA & EA + OTC & EA & EA OTC \\
\cline { 2 - 4 } Temperatura media aire $\left({ }^{\circ} \mathrm{C}\right)$ & $8 \pm 0.3^{\mathrm{a}}$ & $10.2 \pm 0.3^{\mathrm{b}}$ & $4.4 \pm 0.5^{\mathrm{a}}$ & $7.5 \pm 0.6^{\mathrm{b}}$ \\
$\Psi \mathrm{H}_{2} \mathrm{O}_{\text {suelo }}(\mathrm{MPa})$ & $-2.3 \pm 0.3^{\mathrm{a}}$ & $-3.6 \pm 0.4^{\mathrm{b}}$ & - & - \\
TEMPORADA 2007-2008 & & & $9.4 \pm 0.5^{\mathrm{b}}$ \\
Temperatura media aire $\left({ }^{\circ} \mathrm{C}\right)$ & $9.3 \pm 0.3^{\mathrm{a}}$ & $12.6 \pm 0.4^{\mathrm{b}}$ & $5.6 \pm 0.3^{\mathrm{a}}$ & $-0.7 \pm 0.4^{\mathrm{a}}$ \\
$\Psi \mathrm{H}_{2} \mathrm{O}_{\text {suelo }}(\mathrm{MPa})$ & $-2.1 \pm 0.2^{\mathrm{a}}$ & $-4.5 \pm 0.5^{\mathrm{b}}$ & $-0.3 \pm 0.2^{\mathrm{a}}$ & \\
$\mathrm{TEMPORADA} 2008-2009$ & & & \\
Temperatura media aire $\left({ }^{\circ} \mathrm{C}\right)$ & $9.9 \pm 0.3^{\mathrm{a}}$ & $13.2 \pm 0.5^{\mathrm{b}}$ & $7.1 \pm 0.3^{\mathrm{a}}$ & $10.9 \pm 0.6^{\mathrm{b}}$ \\
$\Psi \mathrm{H}_{2} \mathrm{O}_{\text {suelo }}(\mathrm{MPa})$ & $-1.5 \pm 0.9^{\mathrm{a}}$ & $-3.5 \pm 0.6^{\mathrm{b}}$ & $-0.3 \pm 0.2^{\mathrm{a}}$ & $-0.7 \pm 0.4^{\mathrm{a}}$ \\
\hline
\end{tabular}


Adicionalmente, el IRGA entrega variables como la temperatura foliar ( $\mathrm{Tf}$ ) y la densidad de flujo fotónico (DFF), las cuales también fueron registradas e incluidas en la Figura 1.

\section{AnÁLisis ESTADístico}

Para evaluar la significancia de las diferencias del intercambio gaseoso y del microclima en plantas de Phacelia secunda creciendo en distintas condiciones experimentales, se realizaron ANOVAs de dos vías. Las variables predictoras fueron la condición experimental (EA vs. OTC) y la altitud (2900 m vs. $3600 \mathrm{~m})$. Las variables respuesta fueron la tasa de asimilación de $\mathrm{CO}_{2}$, conductancia estomática, temperatura foliar, densidad de flujo fotónico y potencial hídrico de suelo y xilemático. Previo a los ANOVAS los datos fueron chequeados que cumplieran con una distribución normal y homogeneidad de varianza con las pruebas de Shapiro-Wilk y Levene, respectivamente. Los análisis fueron realizados con el software Statistica 6.0 (StatSoft 1999).

\section{RESULTADOS}

\section{PotenCIAL HÍDRICO DEL SUELO Y XILEMÁTICO}

A $2900 \mathrm{~m}$, los $\Psi_{2} \mathrm{O}_{\text {suelo }}$ fueron 2,4 MPa más bajos (i.e. más negativos) dentro de OTC que en EA (Fig. 1a; $\mathrm{F}_{1,15}=73,32$; $p<0,01)$. En cambio a $3600 \mathrm{~m}$, la OTC no tuvo efectos sobre el $\Psi_{2} \mathrm{O}_{\text {suelo }}\left(\mathrm{F}_{1,15}=1,55 ; p=0,22\right)$, el cual registró valores cercanos a -0,4 MPa en ambas condiciones (Fig. 1a), valores que fueron significativamente mayores que a $2900 \mathrm{~m}\left(\mathrm{~F}_{1,15}\right.$ =260,19; $p<0,01)$.

El $\Psi_{2} \mathrm{O}_{\text {xilemático varió significativamente con el }}$ tratamiento y la altitud (Fig. 2e). A 2900 m, el $\Psi_{2} \mathrm{O}_{\text {xilemático }}$ de las plantas de Phacelia secunda creciendo dentro de las OTCs fueron más bajos que el de las plantas que crecen en EAs $\left(\mathrm{F}_{1,19}=11,17 ; p<0,003\right)$. En contraste, a $3600 \mathrm{~m}$ el $\mathrm{HH}_{2} \mathrm{O}_{\text {xilemático }}$ de las plantas creciendo dentro y fuera de OTC fue similar $\left(\mathrm{F}_{1,19}=1,55 ; p=0,22\right)$.

INTERCAMBIO GASEOSO

La fotosíntesis neta (An) de Phacelia secunda varió entre tratamientos y con la altitud (Fig. 2a). A 2900 m, An fue un $40,7 \%$ mayor en plantas creciendo fuera de OTCs que dentro de ellas $\left(\mathrm{F}_{1,16}=4,6 ; p<0,05\right)$. En contraste, las plantas de $3600 \mathrm{~m}$ mostraron un aumento de un $24,4 \%$ en An cuando crecieron dentro de OTC $\left(\mathrm{F}_{1,16}=39,07 ; p<0,05\right)$. La Gs presentó un patrón similar al de An (Fig. 2b). Así, en plantas de menor altitud, Gs disminuyó un 62,1\% cuando éstas crecieron dentro de OTC $\left(\mathrm{F}_{1,16}=56,53 ; p<0,05\right)$, mientras que a mayor altitud, la Gs aumentó un 40,7\% en plantas creciendo dentro de OTCs $\left(\mathrm{F}_{1,16}=14,85 ; p<0,05\right)$. Las OTCs no tuvieron efectos sobre el uso eficiente del agua (UEA) entre plantas creciendo dentro y fuera de OTC en ambas altitudes (Fig. 2c; $F_{1,16}=0,0 ; p=1,0$ ). Sin embargo, sí hubo efectos de la altitud en dicho parámetro, observándose una mayor EUA en las plantas de menor altitud $\left(\mathrm{F}_{1,16}=\right.$ $118,45 ; p<0,01)$.

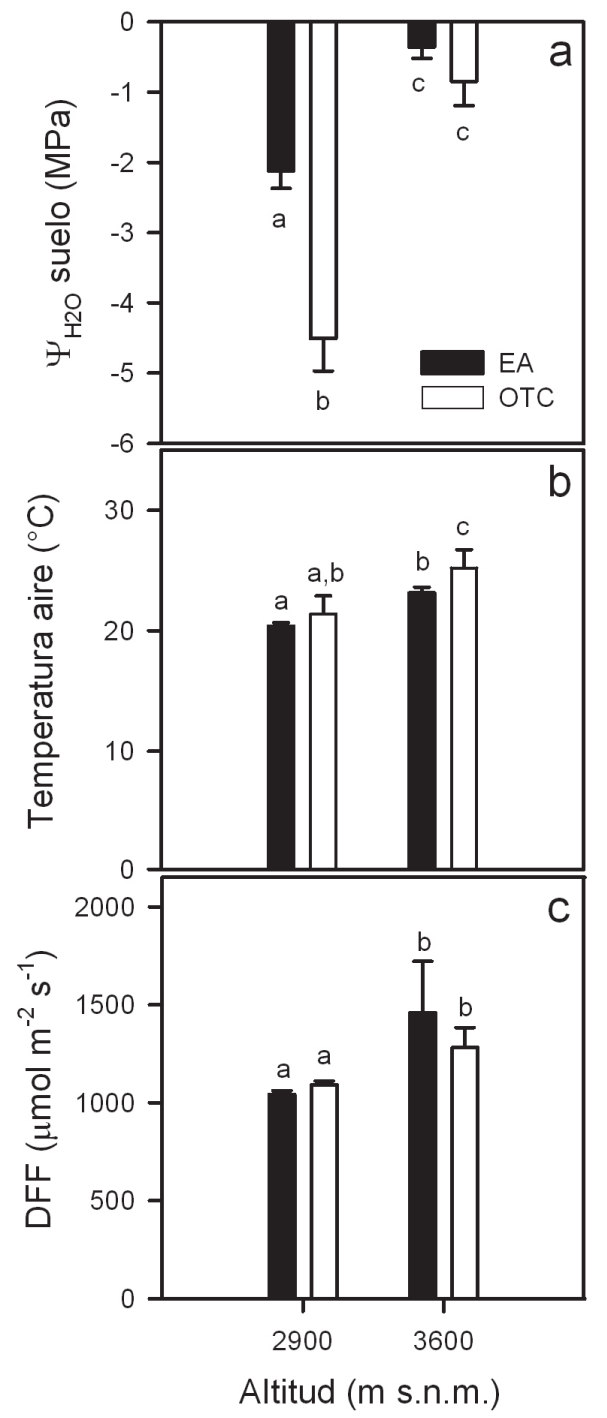

FIgURA 1. Condiciones microclimáticas durante las mediciones de intercambio gaseoso en individuos de Phacelia secunda en dos altitudes de Los Andes de Chile central. Las barras indican valores promedios $\pm \mathrm{EE}(\mathrm{n}=6$, excepto potencial hídrico con $\mathrm{n}=3)$ para: a) potencial hídrico del suelo $(\mathrm{MPa}), \mathrm{\Psi H}_{2} \mathrm{O}_{\text {suelo }}$; b) temperatura del aire $\left({ }^{\circ} \mathrm{C}\right)$ y c) densidad de flujo fotónico $\left(\mu \mathrm{mol} \mathrm{m}^{-2} \mathrm{~s}^{-1}\right)$, DFF. Letras diferentes indican diferencias significativas entre grupos (ANOVA de dos vías; $p<0.05)$.

Figure 1. Microclimatic conditions during gas exchange measurements in Phacelia secunda individuals from two elevations in the central Chilean Andes. Bars correspond to mean values \pm SE ( $n=6$ except by water potential with $n=3$ ) for: a) soil water potential $(\mathrm{MPa}), \mathrm{\Psi H}_{2} \mathrm{O}_{\text {soil }}$; b) air temperature $\left({ }^{\circ} \mathrm{C}\right)$, and c) photon flux density $\left(\mu \mathrm{mol} \mathrm{m} \mathrm{m}^{-2} \mathrm{~s}^{-1}\right)$, DFF. Lower-case letters indicate significant differences between groups (two way ANOVA; $p$ $<0.05)$. 
Las temperaturas foliares (Tf) registradas el día de las mediciones de intercambio gaseoso fueron levemente más altas en plantas creciendo a $3600 \mathrm{~m}$ que a $2900 \mathrm{~m}\left(\mathrm{~F}_{1,20}=\right.$ 36,$7 ; p<0,01$; Fig. 2d). No obstante, no hubo diferencias significativas en $\mathrm{Tf}$ entre las condiciones experimentales a $3600 \mathrm{~m}\left(\mathrm{~F}_{1,20}=10,8 ; p=0,07\right)$. Similarmente, los valores de DFF fueron mayores sobre las plantas de mayor altitud $\left(\mathrm{F}_{1,20}\right.$ $=18,19 ; p<0,01 ;$ Fig. $1 \mathrm{c})$, no encontrándose diferencias entre aquéllas dentro y fuera de OTC $\left(\mathrm{F}_{1,20}=0,80 ; p=0,37\right)$.

\section{DISCUSIÓN}

El aumento de la temperatura ambiental tuvo efectos distintos en la fotosíntesis de Phacelia secunda dependiendo de la altitud a la que se evaluó (Fig. 2). Mientras el aumento de la temperatura ambiental disminuyó el intercambio gaseoso en plantas de $2900 \mathrm{~m}$, tuvo efectos positivos en éste en plantas de $3600 \mathrm{~m}$.

En general, la utilización de OTCs para aumentar la temperatura ambiental tuvo efectos consistentes en las distintas temporadas de crecimiento que se implementó (Tabla I). Así, la temperatura media ambiental fue entre 2,2 y $3,8^{\circ} \mathrm{C}$ más alta dentro de las OTCs en ambas altitudes. Por otro lado, a $2900 \mathrm{~m}$ los potenciales hídricos $\left(\Psi_{2} \mathrm{O}\right)$ del suelo fueron en promedio 1,9 MPa más bajos dentro de las OTC en todas las temporadas. Por lo tanto, la disminución de los $\Psi_{2} \mathrm{O}$ tanto del suelo como xilemáticos registrada durante los días de las mediciones de intercambio gaseoso (Fig. 1a y 2e) son representativas de una condición de menor disponibilidad hídrica para las plantas creciendo a menor altitud y dicha condición se acentúa a mayor temperatura ambiental.

Una de las respuestas más universales de las plantas a la sequía es el cierre estómatico y consecuentemente, una disminución en la conductancia estomática (Farquhar \& Sharkey 1982, Yordanov et al. 2000, Chaves et al. 2009). Esta estrategia es considerada una forma de evasión de la deshidratación, comúnmente presente en plantas anuales y perennes y que tiene como objetivo minimizar la pérdida de agua maximizando la ganancia de carbono (Ehleringer \& Cooper 1992, Chaves et al. 2003). En nuestro estudio, las plantas de menor altitud mostraron una mayor eficiencia en el uso del agua que las de mayor altitud (Fig. 2c). Sin embargo, su tasa de fotosíntesis y conductancia estomática disminuyeron en aquellas plantas expuestas a calentamiento (Fig. 2a y 2b), indicando que el calentamiento podría limitar la capacidad fotosintética de Phacelia secunda, a través de sus efectos negativos sobre la disponibilidad hídrica.

A $3600 \mathrm{~m}$, la fotosíntesis de $P$. secunda estaría limitada principalmente por las bajas temperaturas que se producen por la mayor cantidad de días nublados que se dan en elevaciones altas (sensu Körner 2003). El aumento de la temperatura del aire entre 3,1 y $3,8^{\circ} \mathrm{C}$ generado por las OTCs en esta altitud las temporadas previas (Tabla I) permitió que las plantas al interior de ellas estuvieran expuestas a temperaturas más cercanas a sus óptimos fotosintéticos que las plantas ubicadas en los EAs. Por ejemplo, Cavieres \& Sierra-Almeida (2012) determinaron los "grados días" para el crecimiento de Hordeum comosum dentro y fuera de OTC a $3600 \mathrm{~m}$ (i.e. GDD por su sigla en inglés), como una medida de la cantidad de energía disponible para el crecimiento (MacMaster \& Wilhelm 1997). Los datos microclimáticos de este estudio corresponden a las mismas condiciones experimentales en las que crecieron los individuos de $P$. secunda, mostrando que las plantas creciendo dentro de OTC tuvieron mensualmente $322,1 \pm 90,2^{\circ} \mathrm{C}_{\text {día }}{ }^{-1}$, mientras que aquellas en EAs sólo $165,8 \pm 51{ }^{\circ} \mathrm{C}$ día ${ }^{-1}$ (Cavieres \& Sierra-Almeida 2012). Por lo tanto, las mayores tasas de fotosíntesis y conductancia estomática registradas en plantas creciendo dentro de las OTCs se deberían a mayores GDDs en dichas condiciones. Similares resultados se han encontrado en otras especies de alta montaña (Shaw et al. 2000, Loik et al. 2004, Shi et al. 2010). Por ejemplo, Loik et al. (2004) encontraron que la tasa de fotosíntesis y conductancia estomática del arbusto Artemisia tridentata y de la geófita Erytrhonium grandiflorum fueron mayores bajo condiciones de calentamiento en las montañas Rocosas (Colorado, USA). Sin embargo, otros estudios no han encontrado efectos, o incluso han reportado efectos negativos del calentamiento sobre el desempeño fotosintético de las plantas. Por ejemplo, las herbáceas Erigeron speciosus y Delphinium nutallianum presentaron menores tasas de fotosíntesis y conductancia estomática en condiciones de calentamiento en las Montañas Rocosas (Colorado, USA) (Loik et al. 2000, Lambrecht et al. 2007). Por otro lado, Shi et al. (2010) encontraron efectos contrastantes del calentamiento sobre el desempeño fotosintético de dos especies dominantes del Tibet. En ese estudio, Elymus nutans mostró un aumento de la fotosíntesis y conductancia estomática creciendodentrodeOTCs, encambio Potentilla anserina redujo considerablemente su capacidad fotosintética en condiciones de mayor temperatura. En todos estos estudios, la dirección del efecto del calentamiento sobre la fotosíntesis de las plantas está determinada por sus efectos indirectos sobre la disponibilidad hídrica. Así, cuando el calentamiento disminuyó el potencial hídrico tanto del suelo como de las plantas, los efectos sobre la fotosíntesis fueron negativos (e.g. Loik et al. 2000, Lambrecht et al. 2007). En cambio cuando el aumento de la temperatura ambiental no alteró la disponibilidad hídrica, la mayoría de las plantas respondieron positivamente al calentamiento (Loik et al. 2000, 2004, Lambrecht et al. 2007, Shi et al. 2010). Los resultados obtenidos para Phacelia secunda en este trabajo apuntan en la misma dirección, sugiriendo que los efectos que el aumento de la temperatura ambiental tengan sobre la capacidad fotosintética de las plantas de alta montaña van a depender de sus efectos indirectos sobre la disponibilidad hídrica. 


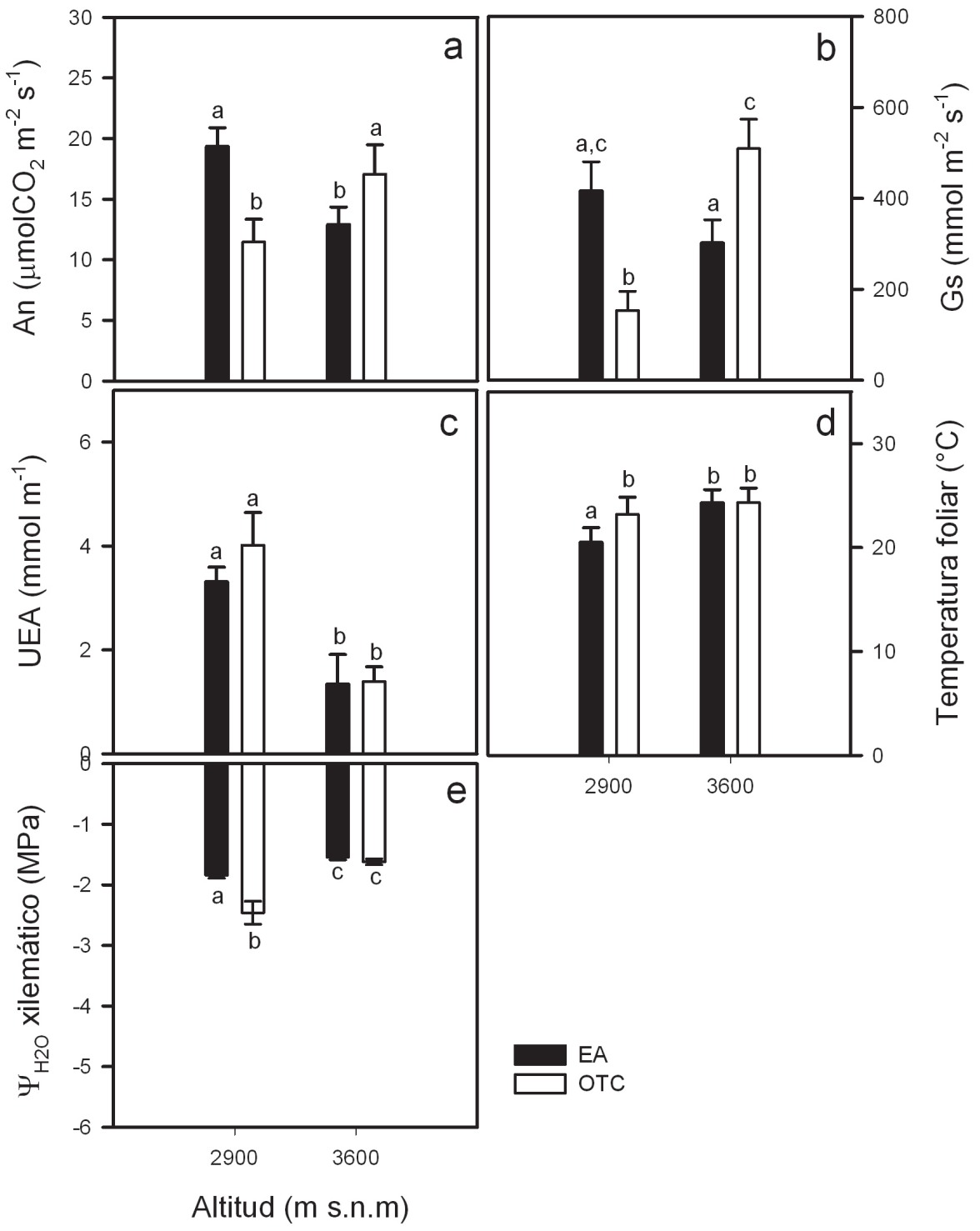

Figura 2. Parámetros fisiológicos medidos en individuos de Phacelia secunda expuestos a un aumento experimental de la temperatura ambiental en dos altitudes de Los Andes de Chile central. Las barras muestran valores promedios \pm EE $(n=6)$ para: a) tasa de asimilación de $\mathrm{CO}_{2}\left(\mu \mathrm{mol} \mathrm{m} \mathrm{m}^{-2} \mathrm{~s}^{-1}\right)$, An; b) Conductancia estomática, $\left(\mathrm{mmol} \mathrm{m} \mathrm{m}^{-2} \mathrm{~s}^{-1}\right)$, Gs; c) Uso eficiente del agua $\left(\mathrm{mmol} \mathrm{m}^{-1}\right)$, UEA; d) temperatura foliar $\left({ }^{\circ} \mathrm{C}\right)$ y e) potencial hídrico xilemático $(\mathrm{MPa}), \Psi_{2} \mathrm{H}_{2} \mathrm{O}_{\text {xilemático }}$. Letras diferentes indican diferencias significativas entre grupos (ANOVA de dos vías; $p<0,05)$.

Figure 2. Physiological parameters measured in individuals of Phacelia secunda exposed to an experimental increase of temperature at two elevations in the central Chilean Andes. Bars show mean values $\pm \mathrm{SE}(\mathrm{n}=6)$ a) $\mathrm{CO}_{2}$ assimilation rate $\left(\mu \mathrm{mol} \mathrm{m}^{-2} \mathrm{~s}^{-1}\right)$, An; b) stomatal conductance $\left(\mathrm{mmol} \mathrm{m} \mathrm{m}^{-2} \mathrm{~s}^{-1}\right)$, Gs; c) water use efficiency $\left(\mathrm{mmol} \mathrm{m} \mathrm{m}^{-1}\right)$, WUE; d) leaf temperature $\left({ }^{\circ} \mathrm{C}\right)$, Tf; and e) xylem water potential $(\mathrm{MPa}), \mathrm{\Psi H}_{2} \mathrm{O}_{\text {xylem }}$. Lower-case letters indicate significant differences among groups (Two way ANOVAs; $p<0.05$ ).

A pesar de que las temperaturas del aire y foliar, y la densidad de flujo fotónico fueron mayores en las plantas de mayor altitud durante las horas de medición de intercambio gaseoso, tanto la fotosíntesis como la conductancia estomática fueron menores a las registradas en plantas de menor altitud. Este patrón invita a discutir dos puntos. Primero, las limitaciones que tienen las variables microclimáticas obtenidas de mediciones instantáneas. Por ejemplo, las mayores temperaturas del aire registradas con el IRGA no coinciden con el patrón de una disminución de la temperatura ambiental con un aumento de la altitud registrada en las distintas temporadas (Tabla I). Esto sugiere que días microclimáticamente atípicos pueden ocurrir, pero no afectan considerablemente el desempeño de las 
plantas. Segundo, la menor tasa fotosintética y conductancia estomática de las plantas de $3600 \mathrm{~m}$ parece estar relacionada en gran medida con la ocurrencia de heladas en los días previos a las mediciones de intercambio gaseoso. Por ejemplo, la madrugada previa a las mediciones en plantas de $3600 \mathrm{~m}$ se registró una helada que alcanzó una intensidad de $-3,4^{\circ} \mathrm{C}$ y duró casi $5 \mathrm{~h}$ (Cavieres et al., datos no publicados). Dichas condiciones favorecen la fotoinhibición de las plantas, especialmente durante las primeras horas del día, produciendo una disminución del rendimiento cuántico del PSII a pesar de que en las horas posteriores se registren temperaturas cercanas al óptimo de fotosíntesis (Germino $\&$ Smith 2001).

Aunque los factores limitantes de la fotosíntesis de Phacelia secunda en ambas altitudes parecen claros, la temperatura foliar varió discretamente entre altitudes y con el aumento de la temperatura ambiental (Fig.1b y 2d). Así, la temperatura foliar fue mayor a mayor altitud y la OTC sólo tuvo efectos sobre ésta en plantas de $2900 \mathrm{~m}$, a pesar de que la temperatura ambiental sólo varió a $3600 \mathrm{~m}$, donde fue mayor dentro de las OTCs. Este patrón sugiere que $P$. secunda posee una serie de rasgos y mecanismos morfológicos y fisiológicos que le permiten regular su temperatura foliar y desacoplarse de las condiciones ambientales. Por ejemplo, $P$. secunda va modificando su morfología a través del gradiente altitudinal, e.g. disminuyendo la estatura de la planta, aumentando el tamaño de la roseta, disminuyendo el ángulo de inclinación de sus hojas y aumentando su superposición y la pubescencia (Cavieres 2000). Aunque es prematuro indicarlo con certeza, algunos estudios preliminares sugieren que dichas modificaciones morfológicas están relacionadas con las características ambientales del lugar y ligadas ampliamente con mecanismos de fotoprotección (C. Hernández et al., datos no publicados).

En conclusión, nuestros resultados sugieren que los efectos del aumento de la temperatura ambiental sobre la fotosíntesis de las plantas en los Andes de Chile central serán distintos dependiendo de la altitud, esperándose efectos negativos en altitudes bajas y efectos positivos en altitudes mayores. Los efectos negativos a altitudes menores se darían fundamentalmente por el aumento en los niveles de sequía, lo que alerta respecto a las predicciones que se han realizado para las zonas de baja altitud de Chile central, donde se esperan disminuciones de las precipitaciones durante el presente siglo (CONAMA 2007). No obstante, es precisamente en estas altitudes donde los modelos pierden certeza respecto a las consecuencias del cambio climático ya que hay modelos que proponen aumentos en las precipitaciones de verano para esta zona (CONAMA 2007). Por lo tanto, es necesario continuar con las indagaciones de los posibles efectos del calentamiento global en el desempeño fotosintético de las plantas de alta-montaña bajo distintos escenarios de disponibilidad hídrica.

\section{AGRADECIMIENTOS}

Este estudio fue financiado por FONDECYT 1090389 y por los Proyectos CONICYT PFB-023 y P05-02, de la Iniciativa Científica Milenio del Ministerio de Economía, Fomento y Turismo del Instituto de Ecología y Biodiversidad.

\section{BIBLIOGRAFÍA}

Aerts, R., J. Cornelissen, E. Dorrepaal, R. Logtestijn \& T. Callaghan. 2004. Effects of experimental imposed climate scenarios on flowering phenology and flower production of subarctic bog species. Global Change Biology 10(9): 1599-1609.

Arft, A.M., M.D. Walker, J. Gurevitch, J.M.Alatalo, M.S. BretHarte, M. Dale, M. Diemer, F. Gugerli, G.H.R. Henry, M.H. Jones, R.D. Hollister, I.S. Jonsdottir, K. Laine, E. Levesque, G.M. Marion, U. Molau, P. Molgaard, U. Nordenhall, V. Raszhivin, C.H. Robinson, G. Starr, A. Stenstrom, M. Stenstrom, O. Totland, P.L. Turner, L.J. Walker, P.J. Webber, J.M. Welker \& P.A. Wookey. 1999. Responses of tundra plants to experimental warming: metaanalysis of the international tundra experiment. Ecological Monographs 69: 491-511.

Beniston, M., H.F. Díaz \& R. S. Bradley. 1997. Climatic change at high elevation sites: An overview. Climatic Change 36: 233-251.

Billings, W.D. \& H.A. Mooney. 1968. The ecology of arctic and alpine plants. Biological Reviews 43: 481-529.

BiLlings, W.D. 1974. Adaptations and origins of alpine plants. Arctic and Alpine Research 6: 129-142.

Buiss, L.C. 1971. Arctic and alpine plant life cycles. Annual Review of Ecology and Systematics 2: 405-438.

Buiss, L.C. 1985. Alpine. In: B.F. Chabot \& H.A. Mooney (eds.), Physiological ecology of North American plant communities; pp. 41-65. Chapman and Hall, London, UK.

CAVIERES, L.A. 2000. Variación morfológica de Phacelia secunda J.F.Gmel. (Hydrophyllaceae) a lo largo de un gradiente altitudinal en Chile central. Gayana Botánica 57: 89-96.

Cavieres, L.A. \& A. Sierra-AlmeidA. 2012. Facilitative interactions do not wane with warming at high-elevations in the Andes. Oecologia (aceptado).

Cavieres, L.A., E. Badano, A. Sierra-Almeida, S. GonZÁlez-Gómez \& M. Molina-Montenegro. 2006. Positive interactions between alpine plant species and the nurse cushion plant Laretia acaulis do not increase with elevation in the Andes of central Chile. New Phytologist 169: 59-69.

Cavieres, L.A., E. Badano, A. Sierra-Almeida \& M. MolinaMontenegro. 2007. Microclimatic modifications of cushion plants and their consequences for seedling survival of native and non-native herbaceous species in the High Andes of Central Chile. Arctic, Antarctic, and Alpine Research 39: 229-236.

Chaves, M.M., J.P. Maroco \& J.S. Pereira. 2003. Understanding plant responses to drought -from genes to the whole plant. Functional Plant Biology 30(3): 239-264.

Chaves, M.M., J. Flexas \& C. Pinheiro. 2009. Photosynthesis under drought and salt stress: regulation mechanisms from whole plant to cell. Annals of Botany 103(4): 551-560.

CONAMA. 2007. Estudio de la Variabilidad Climática en Chile para el siglo XXI. Departamento de Geofísica, Universidad 
de Chile.

Díaz, H.F. \& R.S. BRAdLEy. 1997. Temperature variations during the last century at high elevation sites. Climatic Change 36(3-4): 253-279.

Dormann, C.F. \& S.J. Woodin. 2002. Climate change in the Arctic: using plant functional types in a meta-analysis of field experiments. Functional Ecology 16: 4-17.

Ehleringer, J.R. \& T.A. COOPER. 1992. On the role of orientation in reducing photoinhibitory damage in photosynthetic-twig desert shrubs. Plant, Cell and Environment 15: 301-306.

Farquhar, G.D. \& T.D. Sharkey. 1982. Stomatal conductance and photosynthesis. Annual Review of Plant Physiology and Plant Molecular Biology 33: 317-345.

Germino, M.J. \& W.K. Smith. 2001. Relative importance of microhabitat, plant form, and photosynthetic physiology to carbon gain in two alpine herbs. Functional Ecology 15: 243-251.

Henry, G.H.R. \& U. Molau. 1997. Tundra plants and climate change: the International Tundra Experiment (ITEX). Global Change Biology 3(1):1-9.

HobBie, S.E. \& F.S. Chapin. III. 1998. The response of tundra plant biomass, above-ground production, nitrogen, and $\mathrm{CO}_{2}$ flux to experimental warming. Ecology 79: 1526-1544.

IPCC. 2007. Climate Change 2007: The Physical Science Basis. Cambridge University Press, Cambridge, UK and New York, USA. 114 pp.

KöRnER, C. 2000. The alpine life zone under global change. Gayana Botanica 57: 1-17.

KöRnER, C. 2003. Alpine Plant Life (2da ed). Springe Verlag, Berlin, Germany. 344 pp.

KöRner, C. \& M. Diemer. 1987. In situ photosynthetic responses to light, temperature and carbon dioxide in herbaceous plants from low and high altitude. Functional Ecology 1: 179-194.

Körner, C. \& W. LARCher. 1988. Plant life in cold climates. In: S.P. Long \& F.J. Woodward (eds.), Plants and temperature 42: 25-57. Society for Experimental Biology, Cambridge, $\mathrm{UK}$.

Lambers, H., F.S. Chapin III \& T.L. Pons. 2008. Plant physiological Ecology, $2^{\text {nd }}$ Edition. Springer Science+Business Media LLC, New York. 604 pp.

Lambrecht, S.C., M.E. Loik, D.W. Inouye \& J. Harte. 2006. Reproductive and physiological responses to simulated climate warming for four subalpine species. New Phytologist 173: 121-134.

LoIK, M.E., S.P. Redar \& J. Harte. 2000. Photosynthetic responses to a climate-warming manipulation for contrasting meadow species in the Rocky Mountains, Colorado,USA. Functional Ecology 14: 166-175.

LoIK, M.E. \& S.P. ReDAR. 2003. Freezing tolerance and cold acclimation for seedlings of Artemisia tridentata along an elevation gradient. Journal of Arid Environments 54:769782

Loik, M.E., C. Still, T. Huxman \& J. Harte. 2004. In situ photosynthetic freezing tolerance for plants exposed to a global warming manipulation in the Rocky Mountains, Colorado, USA. New Phytologist 162: 331-341.

McMaster, G.S. \& W.W. Wilhelm. 1997. Growing degree-days: one equation, two interpretations. Agricultural and Forest Meteorology 87: 291-300.

Nagy, L. \& G. Grabherr. 2009. The biology of alpine habitats. Oxford University Press. 363 pp.
Nemani, R.R., C.D. Keeling, H. Hashimoto, W.M. Jolly, S.C. Piper, C.J. Tucker, R.B. Myneni \& S.W. Running. 2003. Climate-driven increases in global terrestrial net primary production from 1982 to 1999. Science 300: 1560-1563.

Norby, R., N. Edwards, J. Riggs, A. Charles, S. Wullschlegger \& C. Gunderson 1997. Temperature-controlled opentop chambers for global change research. Global Change Biology 3(3): 259-267.

Ren, F., H. Zhou, X. Zhao, F. Han, L. Shi, J. Duan \& J. Zhao. 2010. Influence of simulated warming using OTC on physiological-biochemical characteristics of Elymus nutans in alpine meadow on Qinghai-Tibetan plateau. Acta Ecologica Sinica 30(3):166-171.

Rustad, L.E., J.L. Campbell, G.M. Marion, R.J. Norby, M.J. Mitchell, A.E. Hartley, J.H.C. Cornelissen, J. GUREVITCH \& GCTE-NEWS. 2001. A meta-analysis of the response of soil respiration, net nitrogen mineralization, and aboveground plant growth to experimental ecosystem warming. Oecologia 126: 543-562.

SantibáÑez, F. \& J.M. Uribe. 1990. Atlas agroclimático de Chile. Regiones VIII y IX. Ediciones Universidad de Chile, Santiago. 99 pp.

Shaw, M.R., M.E. LoIK \& J. HARTE. 2000. Gas exchange and water relations of two Rocky Mountain shrub species exposed to a climate change manipulation. Plant Ecology 146: 197-206.

ShI, F.S., Y. Wu, N. Wu \& P. Luo. 2010. Different growth and physiological responses to experimental warming of two dominant plant species Elymus nutans and Potentilla anserina in an alpine meadow of the eastern Tibetan Plateau. Photosynthetica 48(3): 437-445.

Sierra-Almeida, A., L.A. Cavieres \& L. Bravo. 2009. Freezing resistance varies within the growing season and with elevation in high-Andean species of central Chile. New Phytologist 182: 461-468.

Streb, P., W. Shang, J. Feierabend \& R. Bligny. 1998. Divergent strategies of photoprotection in high-mountain plants. Planta 207: 313-324.

Walker, M.D., C.H. Wahren, R.D. Hollister, G.H.R. Henry, L.E. Ahlquist, J.M. Alatalo, M.S. Bret-Harteh, M.P. Calefh, T.V. Callaghan, A.B. Carroll, H.E. Epstein, I.S. Jónsdóttir, J.A. Klein, B. Magnússon, U. Molau, S.F. Oberbauer, S.P. Rewa, C.H. Robinson, G.R. Shaver, K.N. Suding, C.C. Thompson, A. Tolvanen, Ø. Totland, P.L. Turner, C.E. Tweedie, P.J. Webber \& P.A. Wookey 2006. Plant community responses to experimental warming across the tundra biome. Proceedings of the National Academy of Sciences 103(5): 1342-1346.

Walther, G.R. 2003. Plants in a warmer world. Perspectives in Plant Ecology, Evolution and Systematics 6(3): 169-185.

Walther, G.R., E. Post \& P. Convey. 2002. Ecological responses to recent climate change. Nature 416: 389-395.

Welker, J.M, K.B. Brown \& J.T. Fahnestock. 1999. CO flux in arctic and alpine dry tundra: comparative field responses under ambient and experimentally warmed conditions. Arctic, Antarctic and Alpine Research 31(3): 272-277.

Welker, J.M., J.T. Fahnestock, G.H.R. Henry, K.W. O’Dea \& R.A. Chimner. 2004. $\mathrm{CO}_{2}$ exchange in three Canadian High Arctic ecosystems: response to long-term experimental warming. Global Change Biology 10(12): 1981-1995.

Yordanov, I., V. Velikova \& T.Tsonev. 2000. Plant responses to drought, acclimation, and stress tolerance. Photosynthetica 38: 171-186.

Recibido: 26.12.11

Aceptado: 05.05.12 